Network Working Group

Request for Comments: 4519

Obsoletes: 2256

Updates: $2247,2798,2377$

Category: Standards Track
A. Sciberras, Ed. eB2Bcom June 2006

\title{
Lightweight Directory Access Protocol (LDAP) :
}

Schema for User Applications

Status of This Memo

This document specifies an Internet standards track protocol for the Internet community, and requests discussion and suggestions for improvements. Please refer to the current edition of the "Internet Official Protocol Standards" (STD 1) for the standardization state and status of this protocol. Distribution of this memo is unlimited.

Copyright Notice

Copyright (C) The Internet Society (2006).

Abstract

This document is an integral part of the Lightweight Directory Access Protocol (LDAP) technical specification. It provides a technical specification of attribute types and object classes intended for use by LDAP directory clients for many directory services, such as White Pages. These objects are widely used as a basis for the schema in many LDAP directories. This document does not cover attributes used for the administration of directory servers, nor does it include directory objects defined for specific uses in other documents. 
Table of Contents

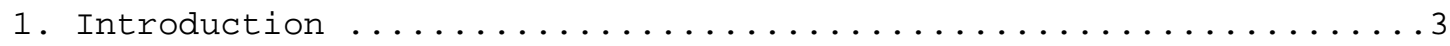

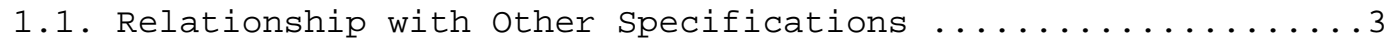

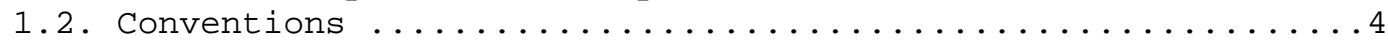

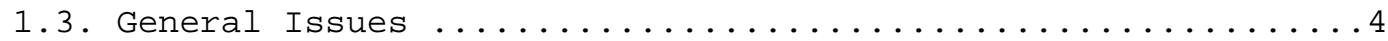

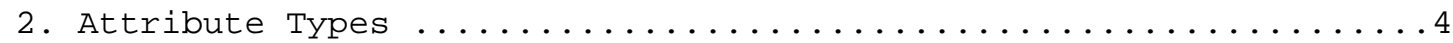

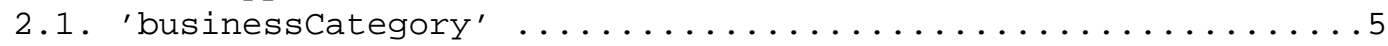

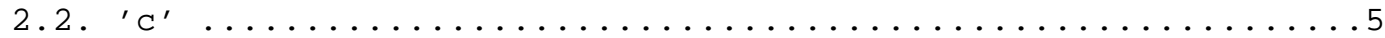

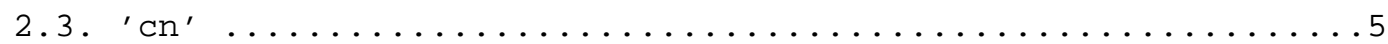

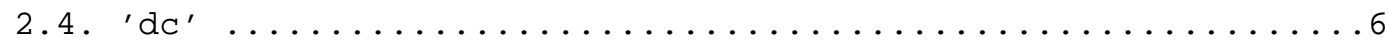

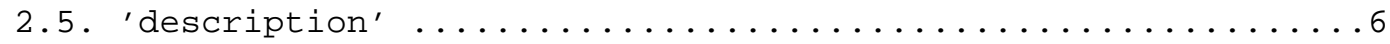

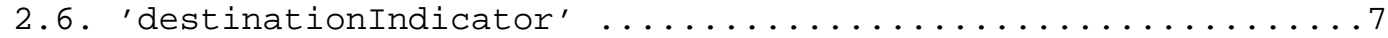

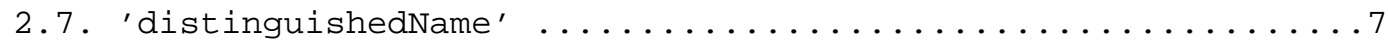

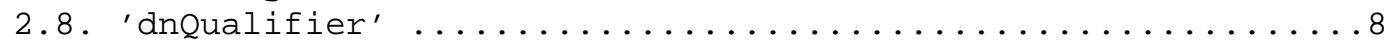

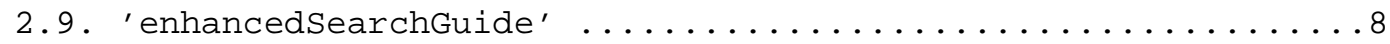

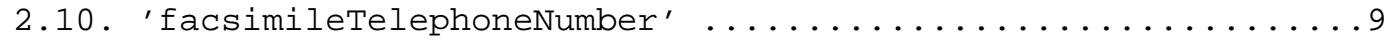

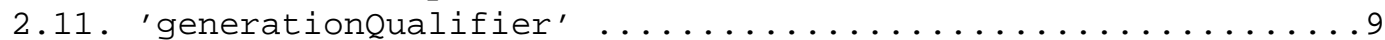

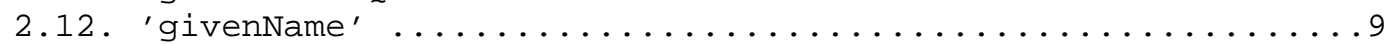

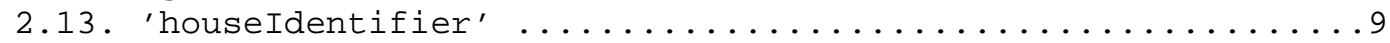

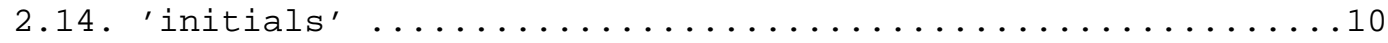

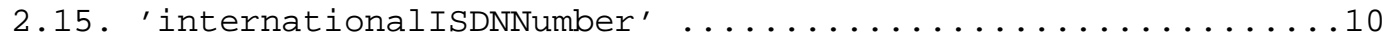

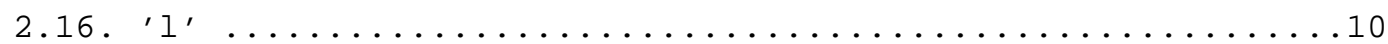

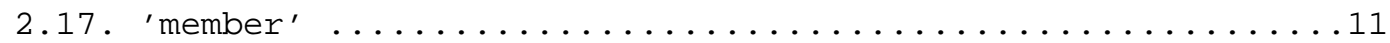

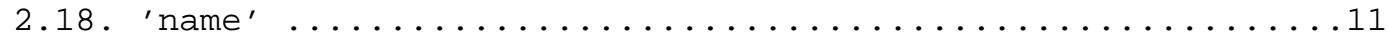

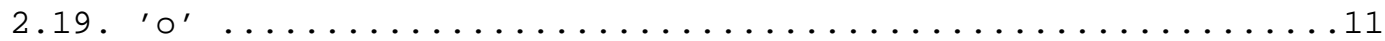

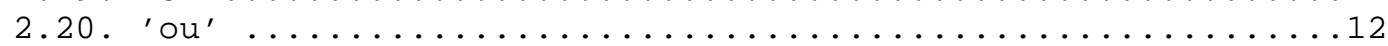

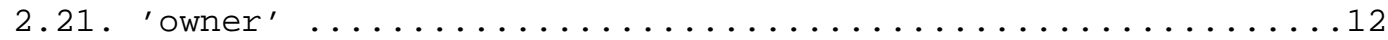

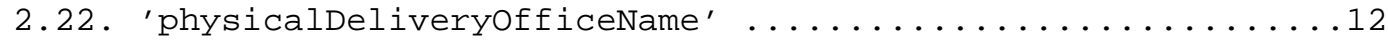

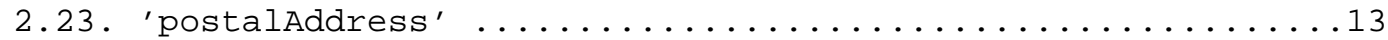

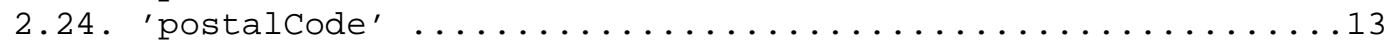

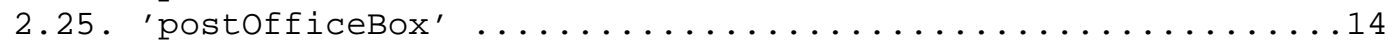

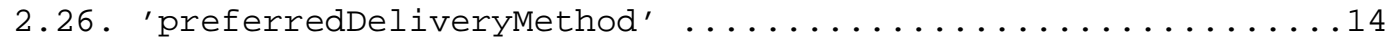

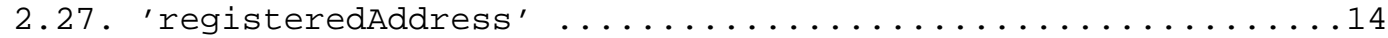

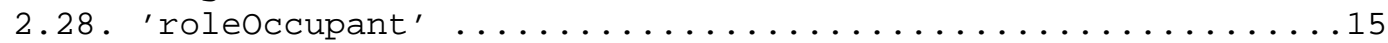

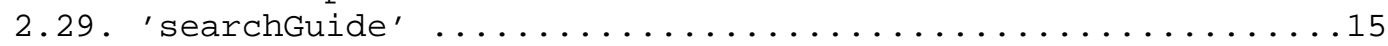

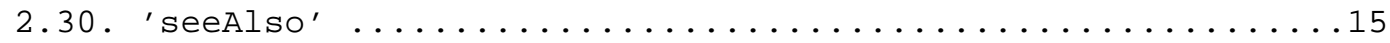

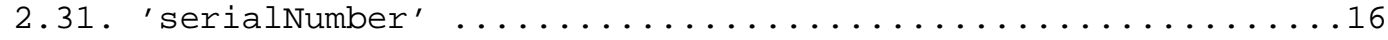

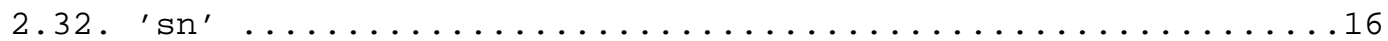

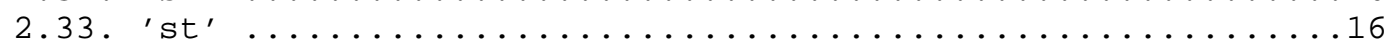

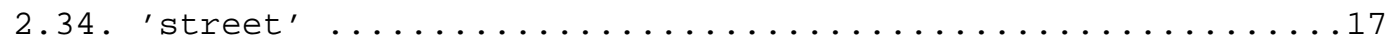

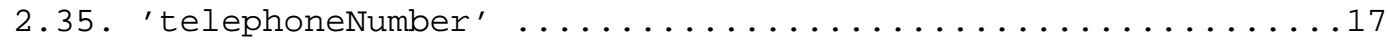

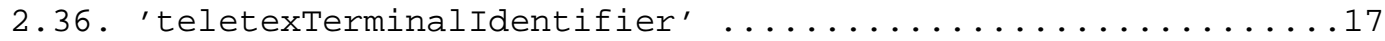

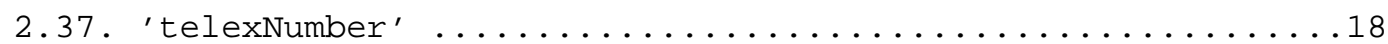

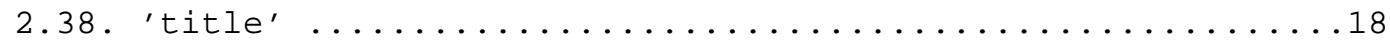

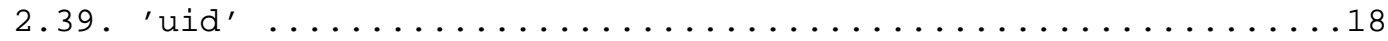

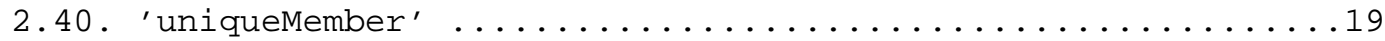

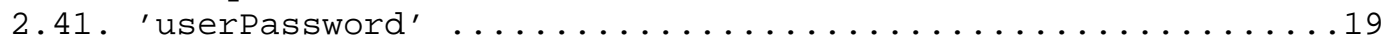




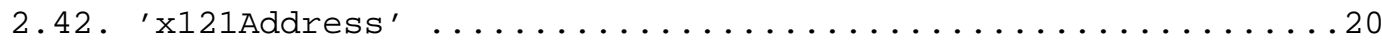

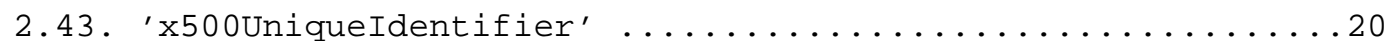

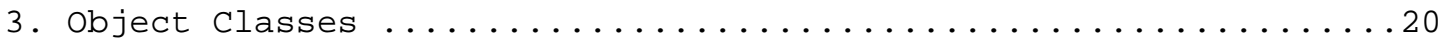

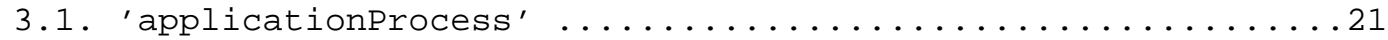

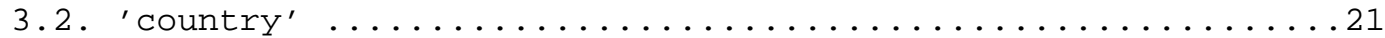

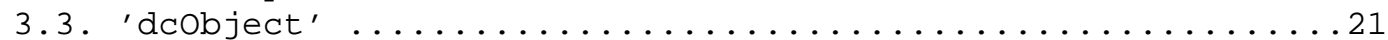

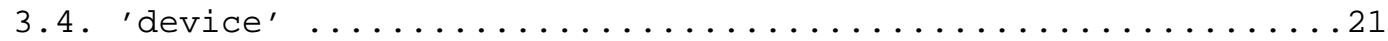

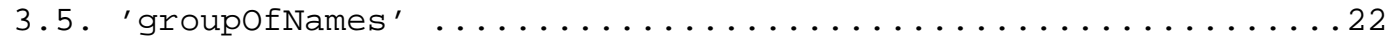

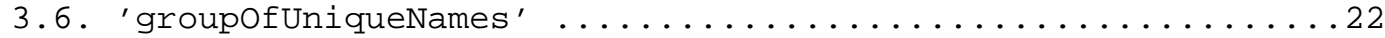

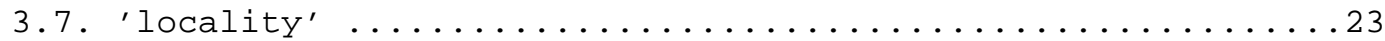

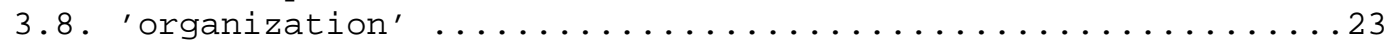

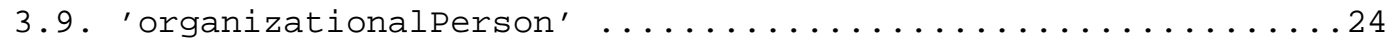

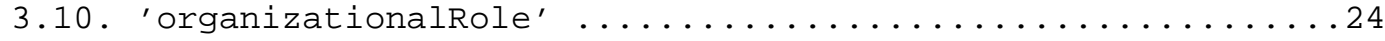

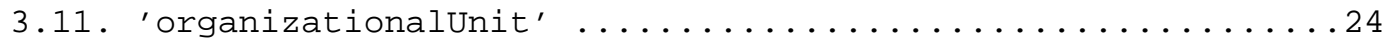

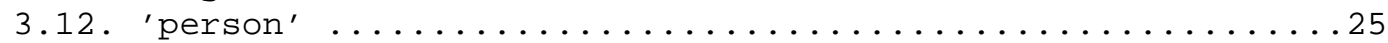

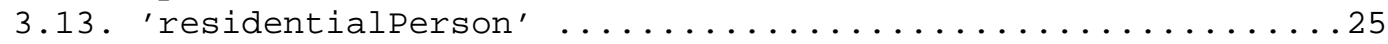

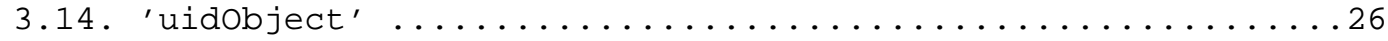

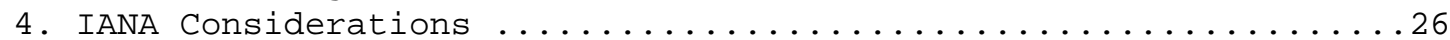

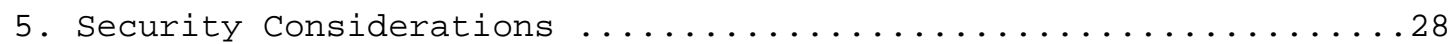

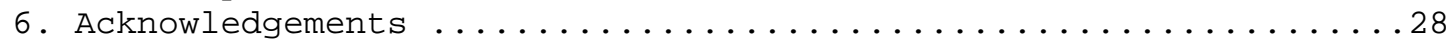

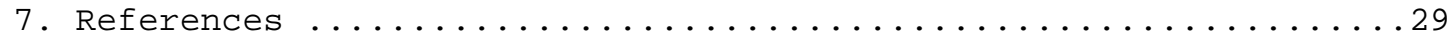

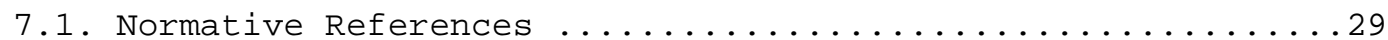

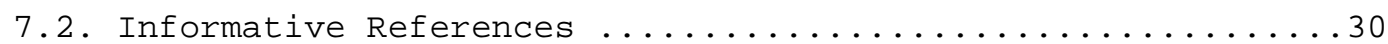

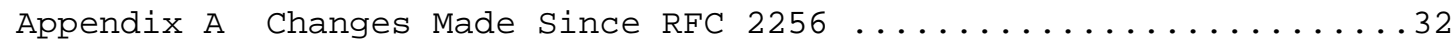

\section{Introduction}

This document provides an overview of attribute types and object classes intended for use by Lightweight Directory Access Protocol (LDAP) directory clients for many directory services, such as white Pages. Originally specified in the X.500 [X.500] documents, these objects are widely used as a basis for the schema in many LDAP directories. This document does not cover attributes used for the administration of directory servers, nor does it include directory objects defined for specific uses in other documents.

\subsection{Relationship with Other Specifications}

This document is an integral part of the LDAP technical specification [RFC4510], which obsoletes the previously defined LDAP technical specification, RFC 3377, in its entirety. In terms of RFC 2256, Sections 6 and 8 of RFC 2256 are obsoleted by [RFC4517]. Sections $5.1,5.2,7.1$, and 7.2 of RFC 2256 are obsoleted by [RFC4512]. The remainder of RFC 2256 is obsoleted by this document. The technical specification for the 'dc' attribute type and 'dcObject' object class found in RFC 2247 are superseded by sections 2.4 and 3.3 of this document. The remainder of RFC 2247 remains in force. 
This document updates RFC 2798 by replacing the informative description of the 'uid' attribute type with the definitive description provided in section 2.39 of this document.

This document updates RFC 2377 by replacing the informative description of the 'uidobject' object class with the definitive description provided in section 3.14 of this document.

A number of schema elements that were included in the previous revision of the LDAP Technical specification are not included in this revision of LDAP. PKI-related schema elements are now specified in [RFC4523]. Unless reintroduced in future technical specifications, the remainder are to be considered Historic.

The descriptions in this document SHALL be considered definitive for use in LDAP.

\subsection{Conventions}

The key words "MUST", "MUST NOT", "REQUIRED", "SHALL", "SHALL NOT", "SHOULD", "SHOULD NOT", "RECOMMENDED", "MAY", and "OPTIONAL" in this document are to be interpreted as described in RFC 2119 [RFC2119].

\subsection{General Issues}

This document references Syntaxes defined in Section 3 of [RFC4517] and Matching Rules defined in Section 4 of [RFC4517].

The definitions of Attribute Types and Object Classes are written using the Augmented Backus-Naur Form (ABNF) [RFC4234] of AttributeTypeDescription and objectClassDescription given in [RFC4512]. Lines have been folded for readability. When such values are transferred as attribute values in the LDAP Protocol, the values will not contain line breaks.

2. Attribute Types

The attribute types contained in this section hold user information.

There is no requirement that servers implement the 'searchGuide' and 'teletexTerminalidentifier' attribute types. In fact, their use is greatly discouraged.

An LDAP server implementation SHOULD recognize the rest of the attribute types described in this section. 


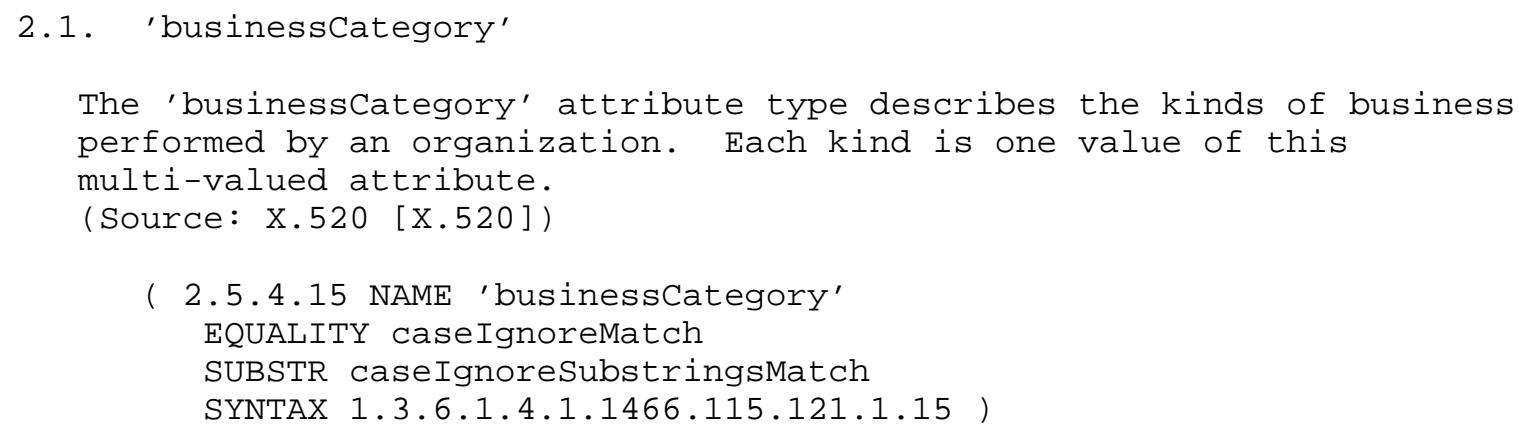

1.3.6.1.4.1.1466.115.121.1.15 refers to the Directory String syntax [RFC 4517].

Examples: "banking", "transportation", and "real estate".

2.2. ' $\mathrm{C}^{\prime}$

The ' $C$ ' ('countryName' in X.500) attribute type contains a two-letter ISO 3166 [ISO3166] country code.

(Source: X.520 [X.520])

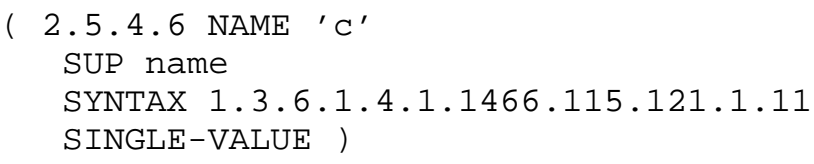

1.3.6.1.4.1.1466.115.121.1.11 refers to the Country String syntax [RFC4517].

Examples: "DE", "AU" and "FR".

\section{3. ' $\mathrm{cn}$ '}

The ' $\mathrm{cn}$ ' ('commonName' in X.500) attribute type contains names of an object. Each name is one value of this multi-valued attribute. If the object corresponds to a person, it is typically the person's full name.

(Source: X.520 [X.520])

( $2.5 .4 .3 \mathrm{NAME}{ }^{\prime} \mathrm{Cn}{ }^{\prime}$ SUP name )

Examples: "Martin K Smith", "Marty Smith" and "printer12". 


\section{4. ' $\mathrm{dc}^{\prime}$}

The 'dc' ('domainComponent' in RFC 1274) attribute type is a string holding one component, a label, of a DNS domain name [RFC1034][RFC2181] naming a host [RFC1123]. That is, a value of this attribute is a string of ASCII characters adhering to the following ABNF [RFC4234]:

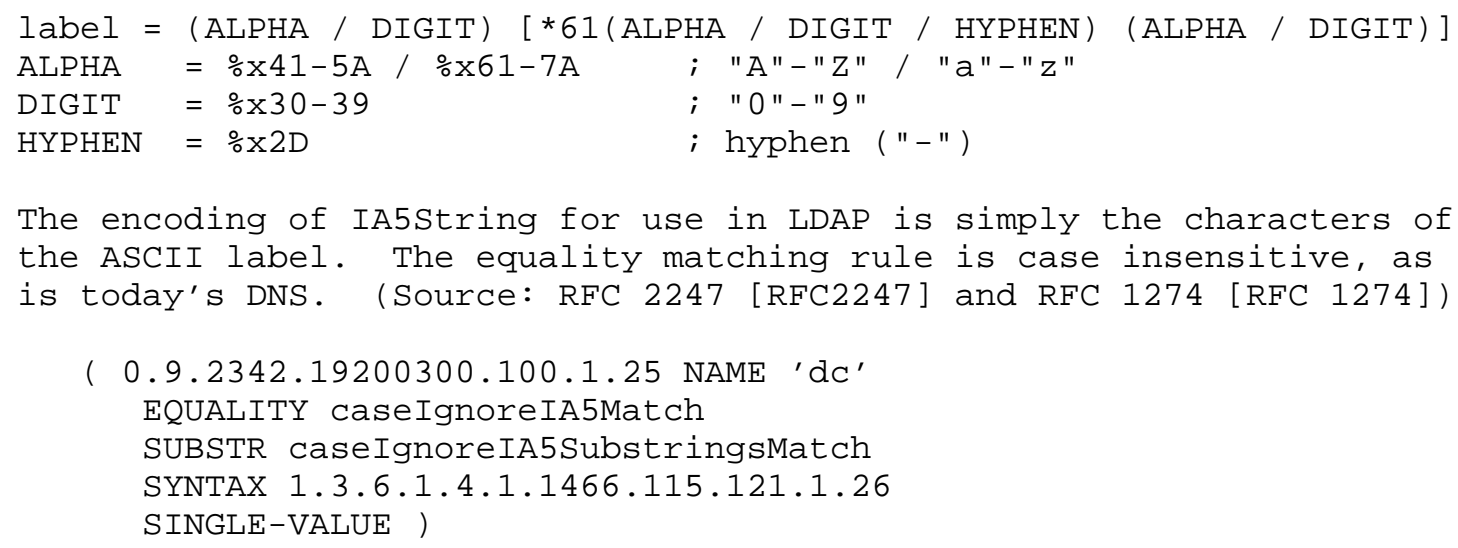




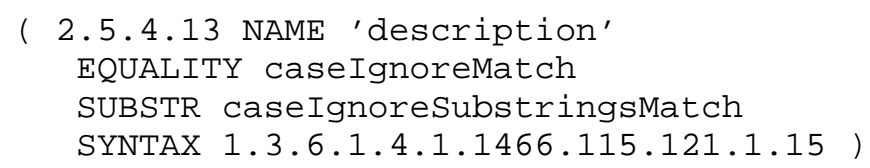

1.3.6.1.4.1.1466.115.121.1.15 refers to the Directory string syntax [RFC4517].

Examples: "a color printer", "Maintenance is done every Monday, at 1pm.", and "distribution list for all technical staff".

\section{6. 'destinationIndicator'}

The 'destinationIndicator' attribute type contains country and city strings associated with the object (the addressee) needed to provide the Public Telegram Service. The strings are composed in accordance with CCITT Recommendations F.1 [F.1] and F.31 [F.31]. Each string is one value of this multi-valued attribute.

(Source: X.520 [X.520])

\section{( 2.5.4.27 NAME 'destinationIndicator' EQUALITY caseIgnoreMatch SUBSTR caseIgnoreSubstringsMatch SYNTAX 1.3.6.1.4.1.1466.115.121.1.44)}

1.3.6.1.4.1.1466.115.121.1.44 refers to the Printable string syntax [RFC4517].

Examples: "AASD" as a destination indicator for Sydney, Australia. "GBLD" as a destination indicator for London, United Kingdom .

It is noted that the directory will not ensure that values of this attribute conform to the F.1 and F.31 CCITT Recommendations. It is the application's responsibility to ensure destination indicators that it stores in this attribute are appropriately constructed.

\section{7. 'distinguishedName'}

The 'distinguishedName' attribute type is not used as the name of the object itself, but it is instead a base type from which some user attribute types with a DN syntax can inherit.

It is unlikely that values of this type itself will occur in an entry. LDAP server implementations that do not support attribute subtyping need not recognize this attribute in requests. Client implementations MUST NOT assume that LDAP servers are capable of performing attribute subtyping. 
(Source: X.520 [X.520])

\section{( 2.5.4.49 NAME 'distinguishedName' EQUALITY distinguishedNameMatch SYNTAX 1.3.6.1.4.1.1466.115.121.1.12)}

1.3.6.1.4.1.1466.115.121.1.12 refers to the DN syntax [RFC4517].

\section{8. 'dnQualifier'}

The 'dnQualifier' attribute type contains disambiguating information strings to add to the relative distinguished name of an entry. The information is intended for use when merging data from multiple sources in order to prevent conflicts between entries that would otherwise have the same name. Each string is one value of this multi-valued attribute. It is recommended that a value of the 'dnQualifier' attribute be the same for all entries from a particular source.

(Source: X.520 [X.520])

\section{( 2.5.4.46 NAME 'dnQualifier' EQUALITY caseIgnoreMatch ORDERING caseIgnoreOrderingMatch SUBSTR caseIgnoreSubstringsMatch SYNTAX 1.3.6.1.4.1.1466.115.121.1.44)}

1.3.6.1.4.1.1466.115.121.1.44 refers to the Printable string syntax [RFC4517].

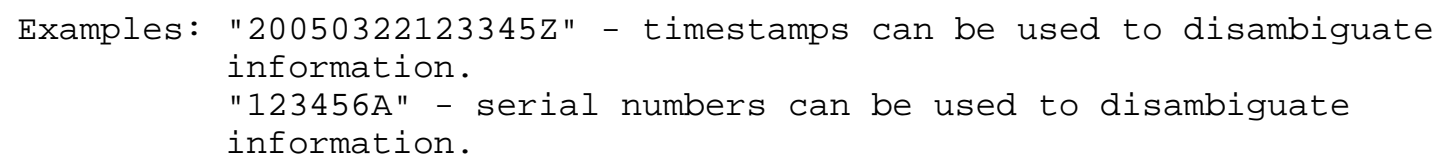

The 'enhancedSearchGuide' attribute type contains sets of information for use by directory clients in constructing search filters. Each set is one value of this multi-valued attribute. (Source: X.520 [X.520])

( 2.5.4.47 NAME 'enhancedSearchGuide' SYNTAX 1.3.6.1.4.1.1466.115.121.1.21)

1.3.6.1.4.1.1466.115.121.1.21 refers to the Enhanced Guide syntax [RFC 4517]. 
Examples: "person\# (sn\$APPROX) \#wholeSubtree" and

"organizationalUnit\# (ou\$SUBSTR) \#oneLevel".

2.10. 'facsimileTelephoneNumber'

The 'facsimileTelephoneNumber' attribute type contains telephone numbers (and, optionally, the parameters) for facsimile terminals. Each telephone number is one value of this multi-valued attribute. (Source: X.520 [X.520])

( 2.5.4.23 NAME 'facsimileTelephoneNumber' SYNTAX 1.3.6.1.4.1.1466.115.121.1.22)

1.3.6.1.4.1.1466.115.121.1.22 refers to the Facsimile Telephone Number syntax [RFC4517].

Examples: "+61 $398967801 "$ and "+81 3347 7418\$fineResolution".

2.11. 'generationQualifier'

The 'generationQualifier' attribute type contains name strings that are typically the suffix part of a person's name. Each string is one value of this multi-valued attribute.

(Source: X.520 [X.520])

\section{( 2.5.4.44 NAME 'generationQualifier'} SUP name )

Examples: "III", "3rd", and "Jr.".

2.12. 'givenName'

The 'givenName' attribute type contains name strings that are the part of a person's name that is not their surname. Each string is one value of this multi-valued attribute.

(Source: X.520 [X.520])

( 2.5.4.42 NAME 'givenName' SUP name )

Examples: "Andrew", "Charles", and "Joanne".

\subsection{3. 'houseIdentifier'}

The 'houseIdentifier' attribute type contains identifiers for a building within a location. Each identifier is one value of this multi-valued attribute. (Source: X.520 [X.520]) 


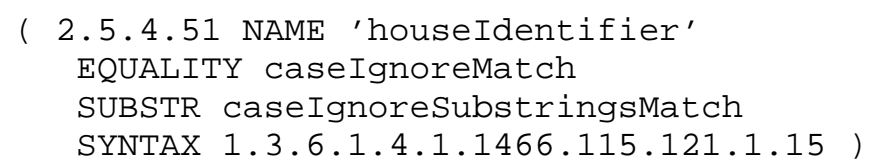

1.3.6.1.4.1.1466.115.121.1.15 refers to the Directory String syntax [RFC4517].

Example: "20" to represent the house number 20 .

2.14. 'initials'

The 'initials' attribute type contains strings of initials of some or all of an individual's names, except the surname(s). Each string is one value of this multi-valued attribute.

(Source: X.520 [X.520])

( 2.5.4.43 NAME 'initials' SUP name )

Examples: "K. A." and "K".

2.15. 'internationalisDNNumber'

The 'internationalISDNNumber' attribute type contains Integrated Services Digital Network (ISDN) addresses, as defined in the International Telecommunication Union (ITU) Recommendation E.164 [E.164]. Each address is one value of this multi-valued attribute. (Source: X.520 [X.520])

\section{( 2.5.4.25 NAME 'internationalISDNNumber' EQUALITY numericStringMatch SUBSTR numericStringSubstringsMatch SYNTAX 1.3.6.1.4.1.1466.115.121.1.36)}

1.3.6.1.4.1.1466.115.121.1.36 refers to the Numeric String syntax [RFC4517].

Example: "0198333 333".

2.16. ' $1{ }^{\prime}$

The 'l' ('localityName' in X.500) attribute type contains names of a locality or place, such as a city, county, or other geographic region. Each name is one value of this multi-valued attribute. (Source: X.520 [X.520]) 


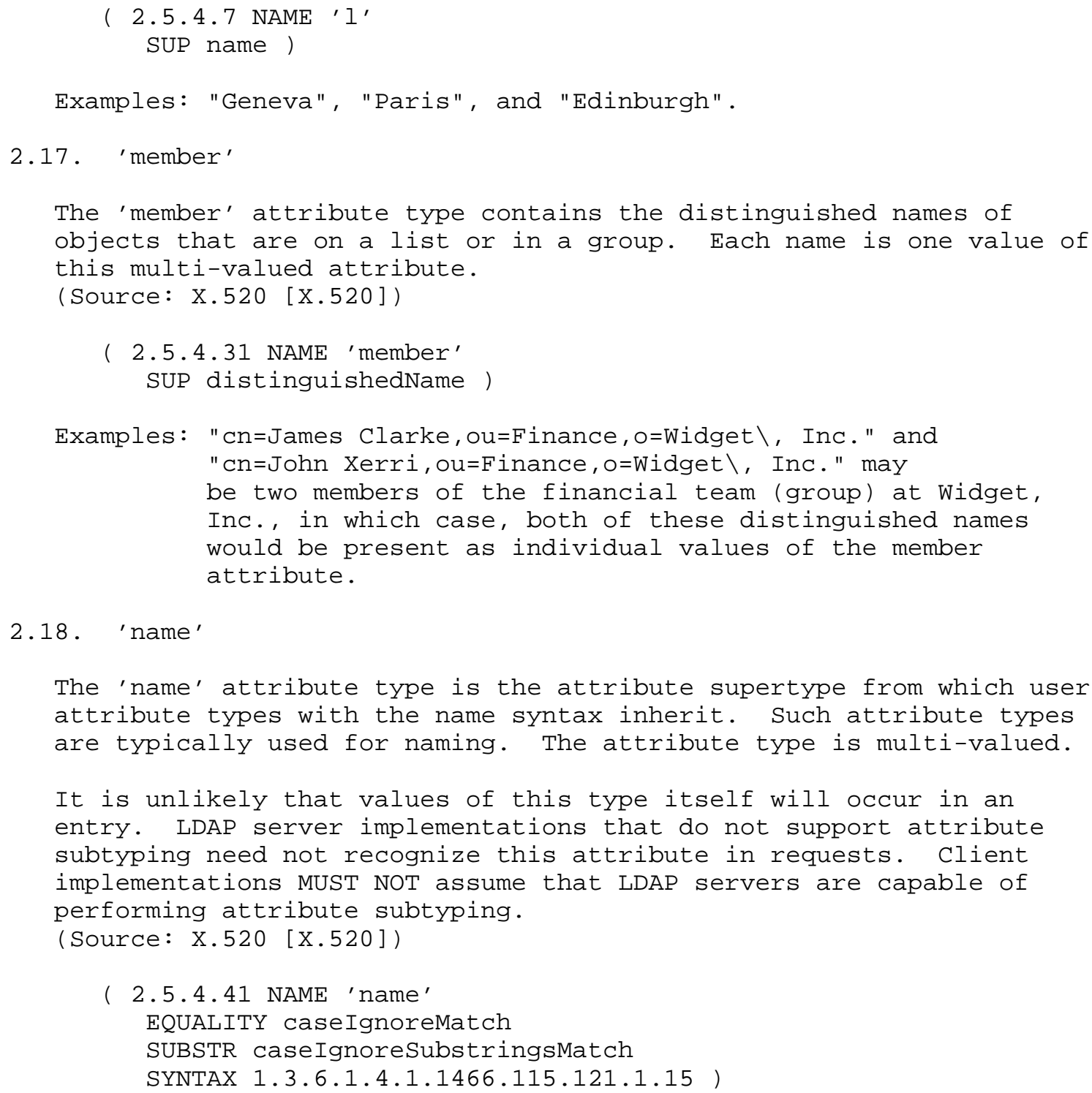


(Source: X.520 [X.520])

( 2.5.4.10 NAME 'o' SUP name )

Examples: "Widget", "Widget, Inc.", and "Widget, Incorporated.".

2.20. 'ou'

The 'ou' ('organizationalUnitName' in X.500) attribute type contains the names of an organizational unit. Each name is one value of this multi-valued attribute.

(Source: X.520 [X.520])

( 2.5.4.11 NAME' 'ou' SUP name )

Examples: "Finance", "Human Resources", and "Research and Development".

\subsection{1. 'owner'}

The 'owner' attribute type contains the distinguished names of objects that have an ownership responsibility for the object that is owned. Each owner's name is one value of this multi-valued attribute.

(Source: X.520 [X.520])

$$
\begin{aligned}
& \text { ( 2.5.4.32 NAME 'owner' } \\
& \text { SUP distinguishedName) }
\end{aligned}
$$

Example: The mailing list object, whose DN is "cn=All Employees, ou=Mailing List, o=Widget $\backslash$, Inc.", is owned by the Human Resources Director.

Therefore, the value of the 'owner' attribute within the mailing list object, would be the DN of the director (role): "cn=Human Resources Director, ou=employee, o=Widget $\backslash$, Inc.".

2.22. 'physicaldeliveryofficeName'

The 'physicalDeliveryofficename' attribute type contains names that a Postal Service uses to identify a post office.

(Source: X.520 [X.520]) 


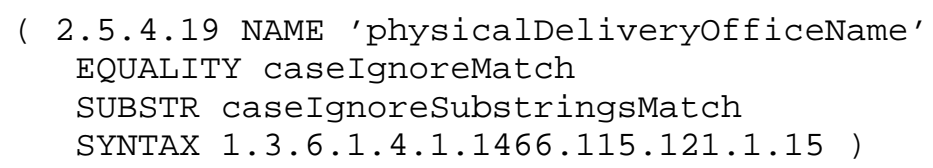

1.3.6.1.4.1.1466.115.121.1.15 refers to the Directory String syntax [RFC4517].

Examples: "Bremerhaven, Main" and "Bremerhaven, Bonnstrasse".

2.23. 'postalAddress'

The 'postalAddress' attribute type contains addresses used by a Postal Service to perform services for the object. Each address is one value of this multi-valued attribute.

(Source: X.520 [X.520])

( 2.5.4.16 NAME 'postalAddress' EQUALITY caseIgnoreListMatch SUBSTR caseIgnoreListSubstringsMatch SYNTAX 1.3.6.1.4.1.1466.115.121.1.41)

1.3.6.1.4.1.1466.115.121.1.41 refers to the Postal Address syntax [RFC 4517].

Example: "15 Main St.\$Ottawa\$Canada".

2.24. 'postalCode'

The 'postalCode' attribute type contains codes used by a Postal Service to identify postal service zones. Each code is one value of this multi-valued attribute.

(Source: X.520 [X.520])

( 2.5.4.17 NAME 'postalCode' EQUALITY caseIgnoreMatch SUBSTR caseIgnoreSubstringsMatch SYNTAX 1.3.6.1.4.1.1466.115.121.1.15)

1.3.6.1.4.1.1466.115.121.1.15 refers to the Directory String syntax [RFC4517].

Example: "22180", to identify Vienna, VA, in the USA. 


\subsection{5. 'postofficeBox'}

The 'postofficeBox' attribute type contains postal box identifiers that a Postal service uses when a customer arranges to receive mail at a box on the premises of the Postal Service. Each postal box identifier is a single value of this multi-valued attribute. (Source: X.520 [X.520])

\section{( 2.5.4.18 NAME 'postofficeBox' EQUALITY caseIgnoreMatch SUBSTR caseIgnoreSubstringsMatch SYNTAX 1.3.6.1.4.1.1466.115.121.1.15)}

1.3.6.1.4.1.1466.115.121.1.15 refers to the Directory String syntax $[\mathrm{RFC} 4517]$.

Example: "Box 45".

2.26. 'preferredDeliveryMethod'

The 'preferredDeliveryMethod' attribute type contains an indication of the preferred method of getting a message to the object.

(Source: X.520 [X.520])

( 2.5.4.28 NAME 'preferredDeliveryMethod' SYNTAX 1.3.6.1.4.1.1466.115.121.1.14 SINGLE-VALUE )

1.3.6.1.4.1.1466.115.121.1.14 refers to the Delivery Method syntax [RFC4517].

Example: If the mhs-delivery Delivery Method is preferred over telephone-delivery, which is preferred over all other methods, the value would be: "mhs \$ telephone".

2.27. 'registeredAddress'

The 'registeredAddress' attribute type contains postal addresses suitable for reception of telegrams or expedited documents, where it is necessary to have the recipient accept delivery. Each address is one value of this multi-valued attribute. (Source: X.520 [X.520])

( 2.5.4.26 NAME 'registeredAddress' SUP postalAddress SYNTAX 1.3.6.1.4.1.1466.115.121.1.41) 
1.3.6.1.4.1.1466.115.121.1.41 refers to the Postal Address syntax [RFC4517].

Example: "Receptionist\$Widget, Inc.\$15 Main St.\$Ottawa\$Canada".

2.28. 'roleoccupant'

The 'roleoccupant' attribute type contains the distinguished names of objects (normally people) that fulfill the responsibilities of a role object. Each distinguished name is one value of this multi-valued attribute.

(Source: X.520 [X.520])

( 2.5.4.33 NAME 'roleoccupant' SUP distinguishedName )

Example: The role object, "cn=Human Resources Director, ou=Position, o=Widget $\backslash$, Inc.", is fulfilled by two people whose object names are "cn=Mary Smith, ou=employee, o=Widget $\backslash$, Inc." and "cn=James Brown, ou=employee, o=Widget $\backslash$, Inc.". The 'roleoccupant' attribute will contain both of these distinguished names, since they are the occupants of this role.

\subsection{9. 'searchGuide'}

The 'searchGuide' attribute type contains sets of information for use by clients in constructing search filters. It is superseded by

'enhancedSearchGuide', described above in Section 2.9. Each set is one value of this multi-valued attribute.

(Source: X.520 [X.520])

( 2.5.4.14 NAME ' searchGuide' SYNTAX 1.3.6.1.4.1.1466.115.121.1.25)

1.3.6.1.4.1.1466.115.121.1.25 refers to the Guide syntax [RFC4517]. Example: "person\#sn\$EQ".

2.30. 'seeAlso'

The 'seeAlso' attribute type contains the distinguished names of objects that are related to the subject object. Each related object name is one value of this multi-valued attribute.

(Source: X.520 [X.520])

( 2.5.4.34 NAME ' seeAlso' SUP distinguishedName ) 
Example: The person object " $\mathrm{cn}=$ James Brown, ou=employee, o=Widget $\backslash$, Inc." is related to the role objects "cn=Football Team Captain, ou=sponsored activities, o=Widget $\backslash$, Inc." and " $\mathrm{cn}=$ Chess Team, ou=sponsored activities, o=Widget $\backslash$, Inc.". Since the role objects are related to the person object, the ' seeAlso' attribute will contain the distinguished name of each role object as separate values.

2.31. 'serialNumber'

The 'serialNumber' attribute type contains the serial numbers of devices. Each serial number is one value of this multi-valued attribute.

(Source: X.520 [X.520])

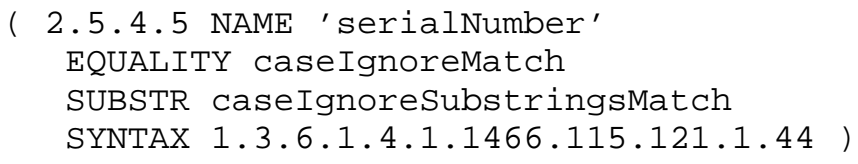

1.3.6.1.4.1.1466.115.121.1.44 refers to the Printable string syntax [RFC4517].

Examples: "WI-3005" and "XF551426".

2.32. ' $\mathrm{sn}^{\prime}$

The 'sn' ('surname' in X.500) attribute type contains name strings for the family names of a person. Each string is one value of this multi-valued attribute.

(Source: X.520 [X.520])

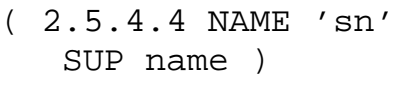

Example: "Smith".

\subsection{3. 'st'}

The 'st' ('stateorprovinceName' in X.500) attribute type contains the full names of states or provinces. Each name is one value of this multi-valued attribute.

(Source: X.520 [X.520])

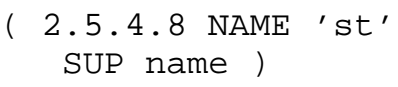

Example: "California". 


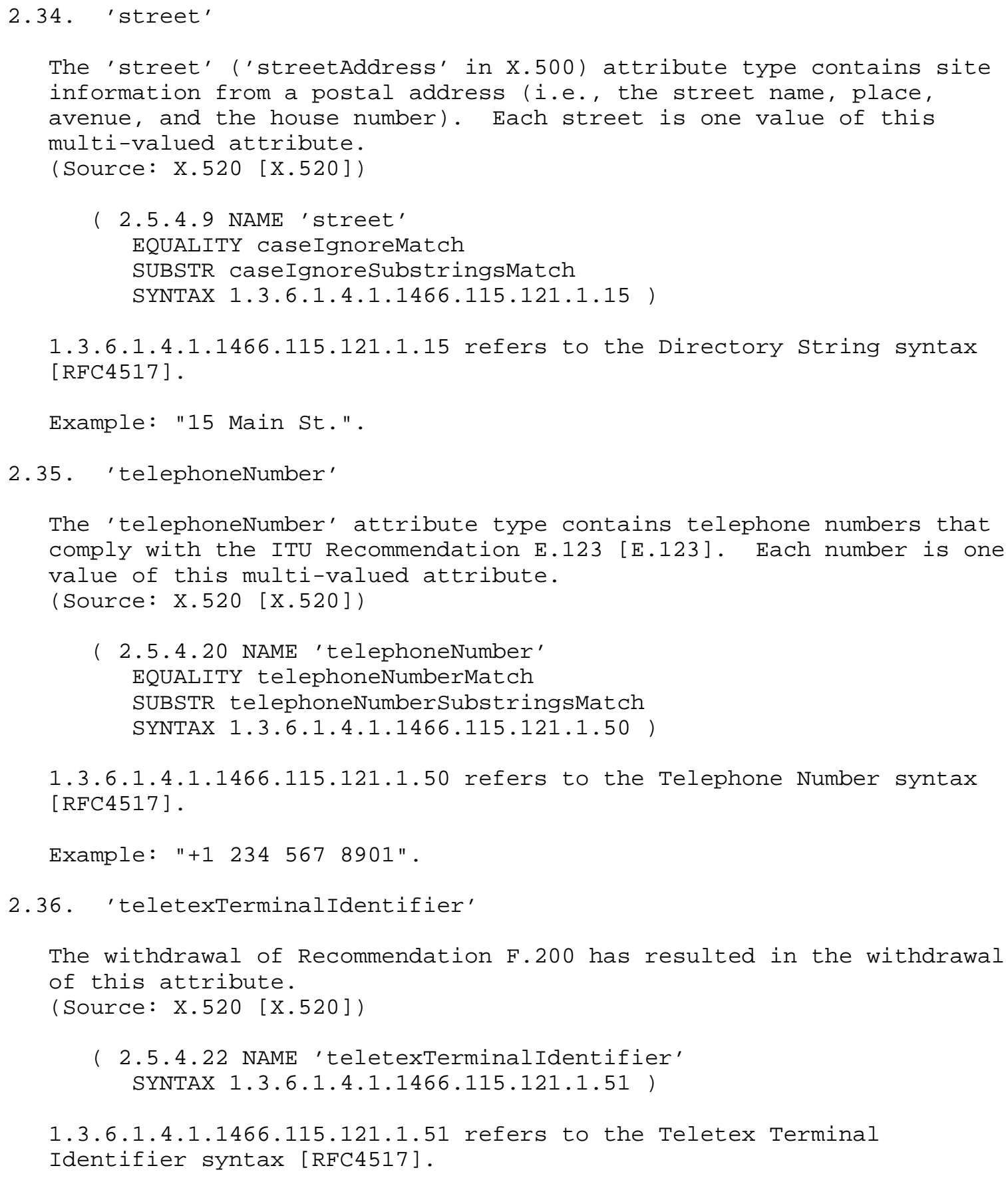




\subsection{7. 'telexNumber'}

The 'telexNumber' attribute type contains sets of strings that are a telex number, country code, and answerback code of a telex terminal. Each set is one value of this multi-valued attribute. (Source: X.520 [X.520])

( 2.5.4.21 NAME 'telexNumber' SYNTAX 1.3.6.1.4.1.1466.115.121.1.52)

1.3.6.1.4.1.1466.115.121.1.52 refers to the Telex Number syntax [RFC4517].

Example: "12345\$023\$ABCDE" .

2.38. 'title'

The 'title' attribute type contains the title of a person in their organizational context. Each title is one value of this multi-valued attribute.

(Source: X.520 [X.520])

( 2.5.4.12 NAME' 'title' SUP name )

Examples: "Vice President", "Software Engineer", and "CEO".

2.39. 'uid'

The 'uid' ('userid' in RFC 1274) attribute type contains computer system login names associated with the object. Each name is one value of this multi-valued attribute.

(Source: RFC 2798 [RFC2798] and RFC 1274 [RFC1274])

( 0.9.2342.19200300.100.1.1 NAME 'uid'

EQUALITY caseIgnoreMatch

SUBSTR caseIgnoreSubstringsMatch

SYNTAX 1.3.6.1.4.1.1466.115.121.1.15)

1.3.6.1.4.1.1466.115.121.1.15 refers to the Directory String syntax [RFC4517].

Examples: "s9709015", "admin", and "Administrator". 


\subsection{0. 'uniqueMember'}

The 'uniqueMember' attribute type contains the distinguished names of an object that is on a list or in a group, where the relative distinguished names of the object include a value that distinguishes between objects when a distinguished name has been reused. Each distinguished name is one value of this multi-valued attribute. (Source: X.520 [X.520])

\section{( 2.5.4.50 NAME ' uniqueMember' EQUALITY uniqueMemberMatch SYNTAX 1.3.6.1.4.1.1466.115.121.1.34)}

1.3.6.1.4.1.1466.115.121.1.34 refers to the Name and Optional UID syntax [RFC4517].

Example: If "ou=1st Battalion, o=Defense, c=US" is a battalion that was disbanded, establishing a new battalion with the "same" name would have a unique identifier value added, resulting in "ou=1st Battalion, o=Defense, c=US\#'010101'B".

\subsection{1. 'userPassword'}

The 'userpassword' attribute contains octet strings that are known only to the user and the system to which the user has access. Each string is one value of this multi-valued attribute.

The application SHOULD prepare textual strings used as passwords by transcoding them to Unicode, applying SASLprep [RFC4013], and encoding as UTF-8. The determination of whether a password is textual is a local client matter.

(Source: X.509 [X.509])

\section{( 2.5.4.35 NAME 'userPassword' EQUALITY octetstringMatch SYNTAX 1.3.6.1.4.1.1466.115.121.1.40)}

1.3.6.1.4.1.1466.115.121.1.40 refers to the Octet string syntax [RFC4517].

Passwords are stored using an Octet String syntax and are not encrypted. Transfer of cleartext passwords is strongly discouraged where the underlying transport service cannot guarantee confidentiality and may result in disclosure of the password to unauthorized parties.

An example of a need for multiple values in the 'userpassword' attribute is an environment where every month the user is expected to 
use a different password generated by some automated system. During transitional periods, like the last and first day of the periods, it may be necessary to allow two passwords for the two consecutive periods to be valid in the system.

2.42. 'x121Address'

The 'x121Address' attribute type contains data network addresses as defined by ITU Recommendation X.121 [X.121]. Each address is one value of this multi-valued attribute.

(Source: X.520 [X.520])

\section{( 2.5.4.24 NAME 'x121Address' EQUALITY numericStringMatch SUBSTR numericStringSubstringsMatch SYNTAX 1.3.6.1.4.1.1466.115.121.1.36)}

1.3.6.1.4.1.1466.115.121.1.36 refers to the Numeric String syntax [RFC4517].

Example: "36111222333444555".

2.43. 'x500UniqueIdentifier'

The 'x500UniqueIdentifier' attribute type contains binary strings that are used to distinguish between objects when a distinguished name has been reused. Each string is one value of this multi-valued attribute.

In X.520 [X.520], this attribute type is called 'uniqueIdentifier'. This is a different attribute type from both the 'uid' and ' uniqueIdentifier' LDAP attribute types. The 'uniqueIdentifier' attribute type is defined in [RFC4524].

(Source: X.520 [X.520])

( 2.5.4.45 NAME 'x500UniqueIdentifier' EQUALITY bitstringMatch SYNTAX 1.3.6.1.4.1.1466.115.121.1.6)

1.3.6.1.4.1.1466.115.121.1.6 refers to the Bit string syntax [RFC4517].

3. Object Classes

LDAP servers SHOULD recognize all the Object Classes listed here as values of the 'objectClass' attribute (see [RFC4512]). 


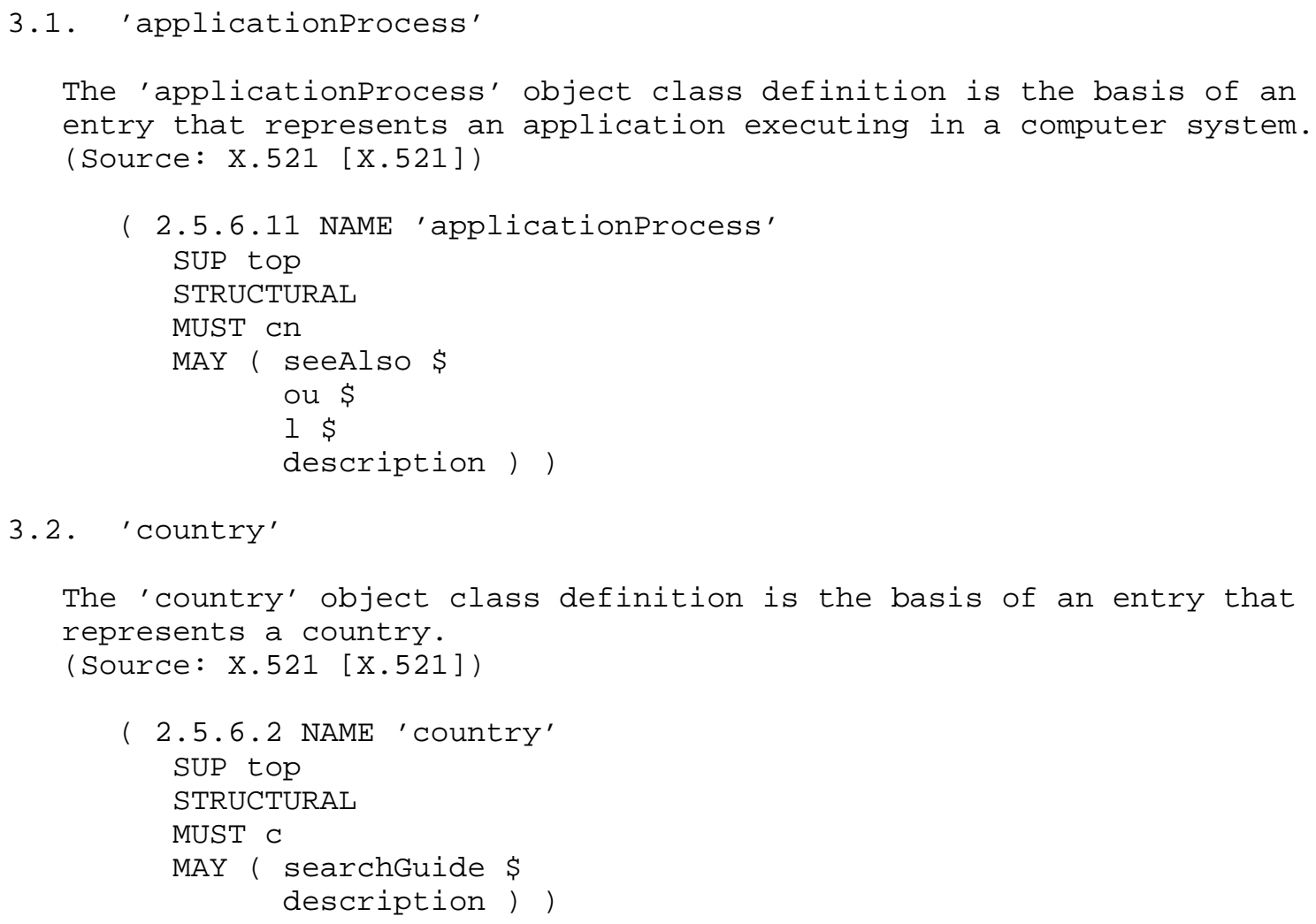




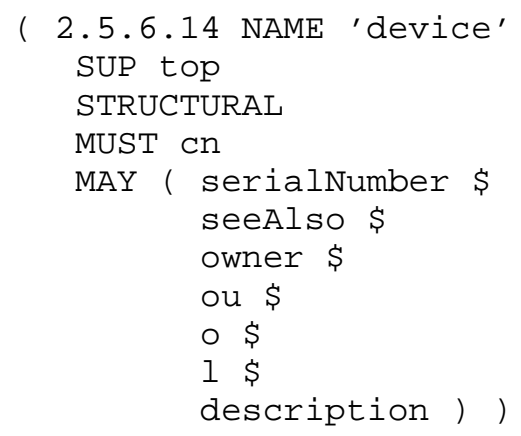

\section{5. 'groupofNames'}

The 'groupofNames' object class is the basis of an entry that represents a set of named objects including information related to the purpose or maintenance of the set.

(Source: X.521 [X.521])

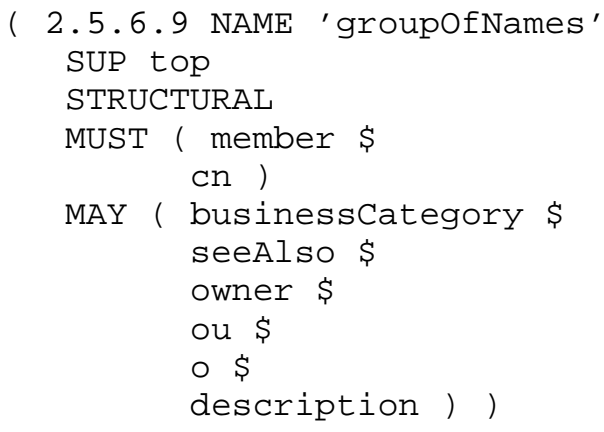

3.6. 'groupofUniqueNames'

The 'groupofuniqueNames' object class is the same as the

'groupofNames' object class except that the object names are not repeated or reassigned within a set scope.

(Source: X.521 [X.521]) 


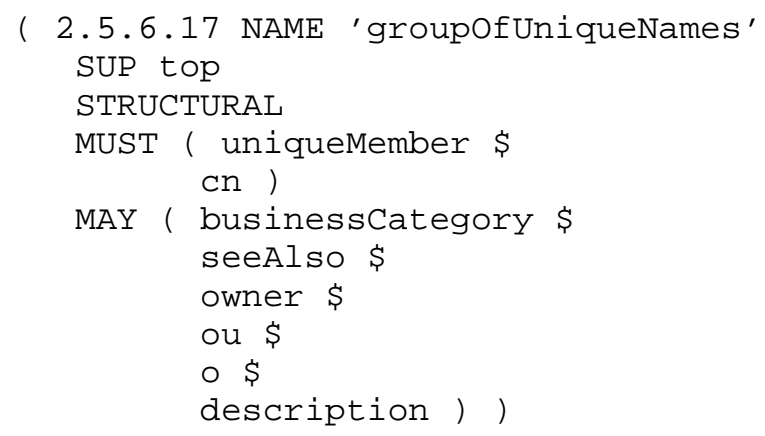

\section{7. 'locality'}

The 'locality' object class is the basis of an entry that represents a place in the physical world. (Source: X.521 [X.521])

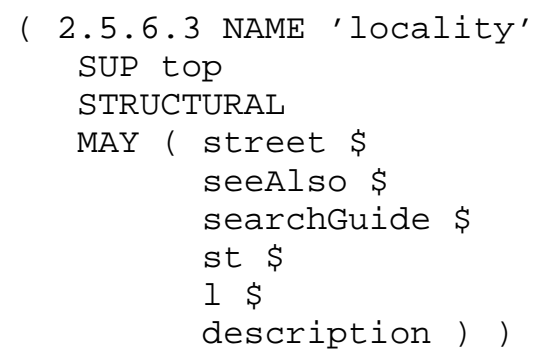

\section{8. 'organization'}

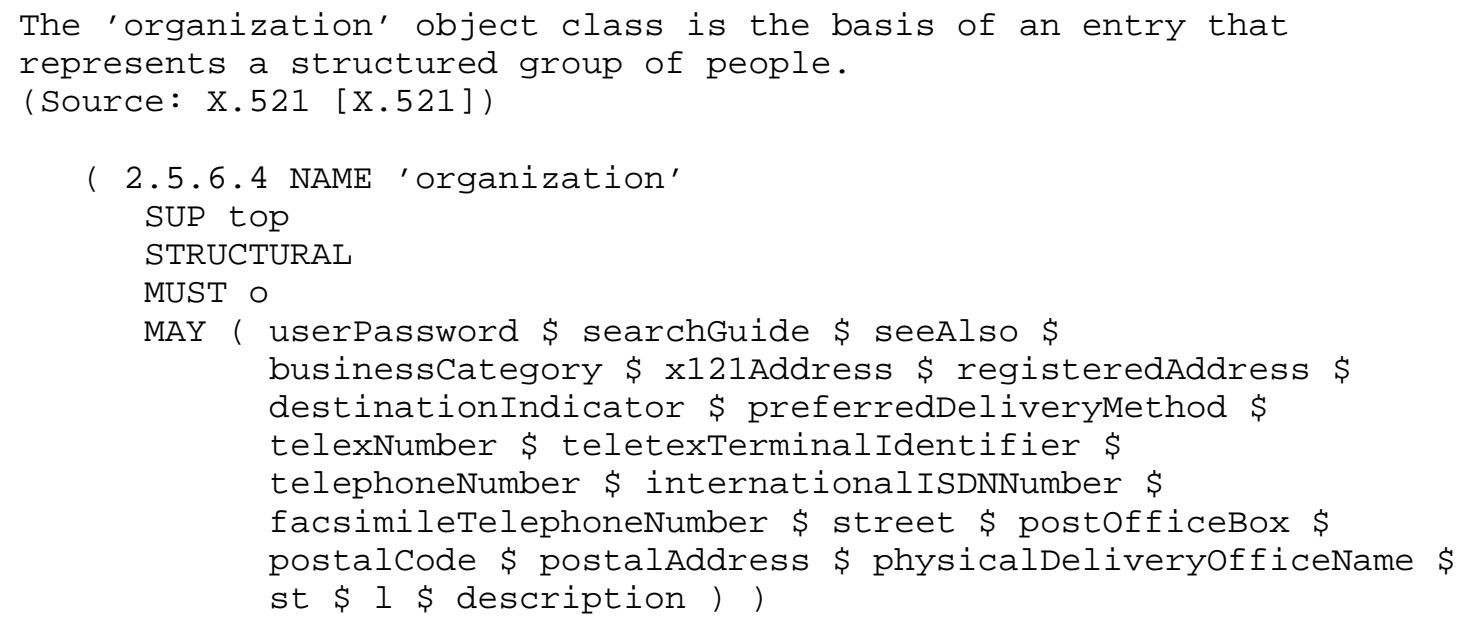




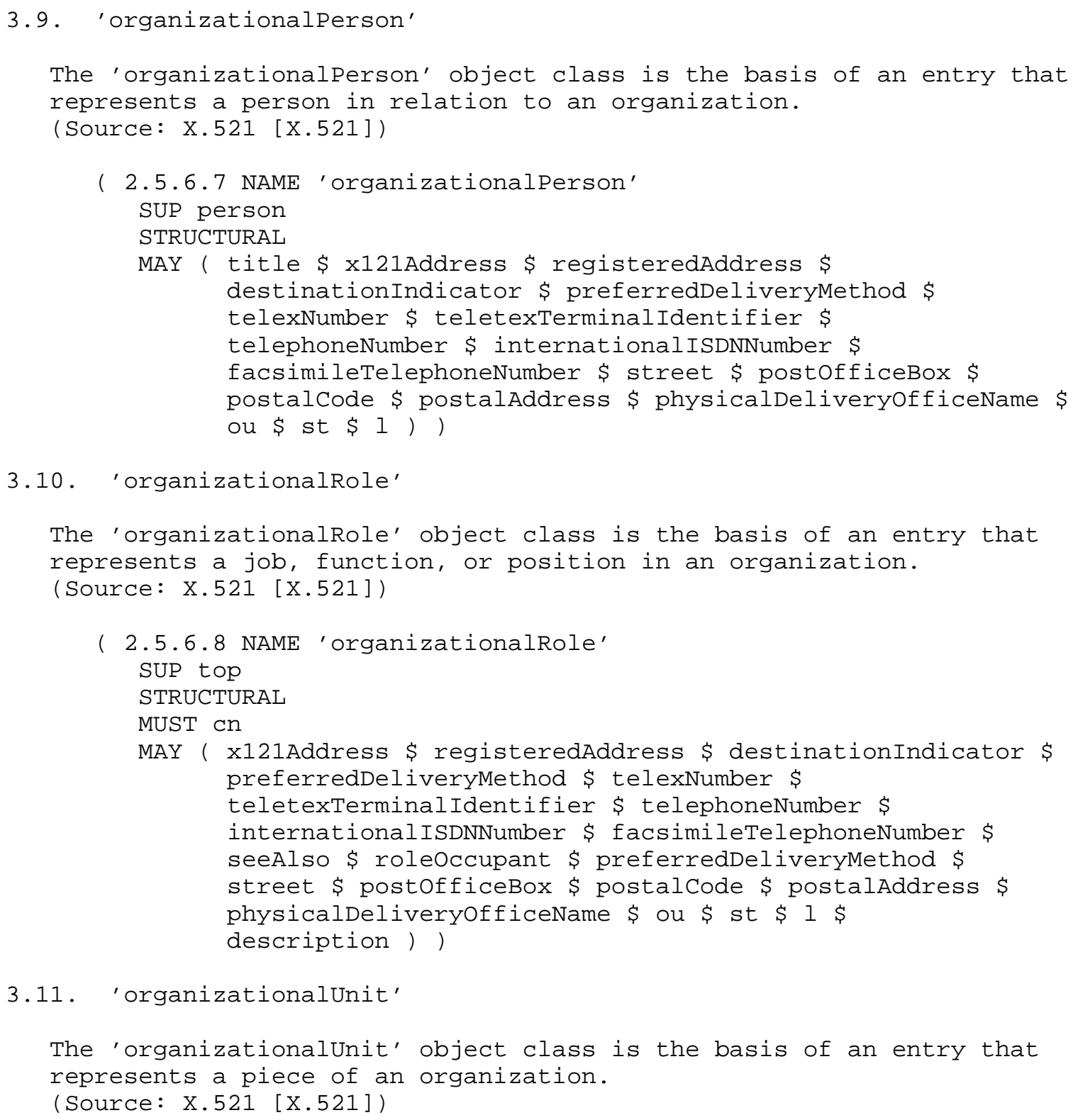




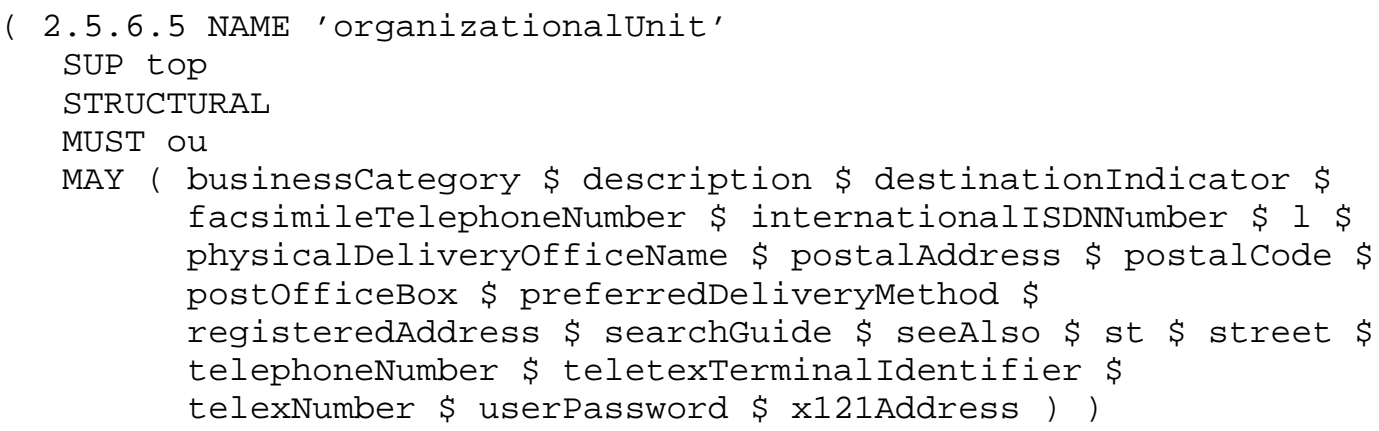

\subsection{2 'person'}

The 'person' object class is the basis of an entry that represents a human being.

(Source: X.521 [X.521])

( 2.5.6.6 NAME 'person'

SUP top STRUCTURAL

MUST ( sn \$

(n)

MAY ( userPassword \$ telephoneNumber $\$$ seeAlso \$ description ) )

3.13. 'residentialPerson'

The 'residentialperson' object class is the basis of an entry that includes a person's residence in the representation of the person. (Source: X.521 [X.521])

( 2.5.6.10 NAME 'residentialperson'

SUP person

STRUCTURAL

MUST 1

MAY ( businessCategory $\$$ xi21Address $\$$ registeredAddress $\$$ destinationIndicator $\$$ preferredDeliveryMethod \$ telexNumber \$ teletexTerminalidentifier \$ telephoneNumber $\$$ internationalisDNNumber $\$$ facsimileTelephoneNumber $\$$ preferredDeliveryMethod $\$$ street $\$$ postofficeBox $\$$ postalcode $\$$ postalAddress $\$$ physicaldeliveryofficeName $\$$ st $\$ 1$ ) ) 


\subsection{4. 'uidobject'}

The 'uidobject' object class permits an entry to contains user identification information. This object class is defined as auxiliary, because it will be used in conjunction with an existing structural object class.

(Source: RFC 2377 [RFC2377])

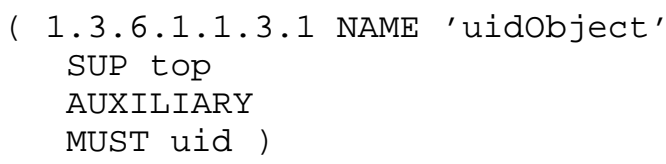

4. IANA Considerations

The Internet Assigned Numbers Authority (IANA) has updated the LDAP descriptors registry as indicated in the following template:

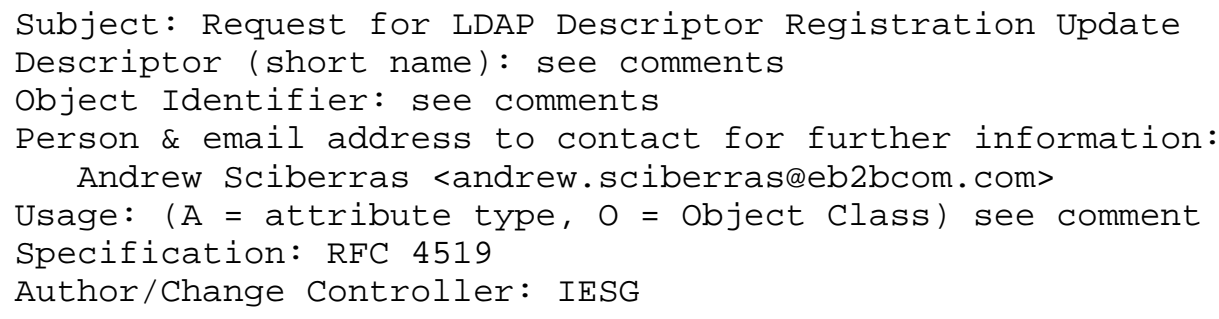

Comments

In the LDAP descriptors registry, the following descriptors (short names) have been updated to refer to RFC 4519. Names that need to be reserved, rather than assigned to an object Identifier, will contain an Object Identifier value of RESERVED.

\begin{tabular}{|c|c|c|}
\hline NAME & Type & OID \\
\hline applicationProcess & 0 & 2.5 .6 .11 \\
\hline businessCategory & A & 2.5 .4 .15 \\
\hline $\mathrm{C}$ & A & 2.5 .4 .6 \\
\hline $\mathrm{Cn}$ & A & 2.5 .4 .3 \\
\hline commonName & A & 2.5 .4 .3 \\
\hline country & O & 2.5 .6 .2 \\
\hline countryName & $\mathrm{A}$ & 2.5 .4 .6 \\
\hline de & A & 0.9 .2342 .19200300 .100 .1 .25 \\
\hline dcObject & O & 1.3 .6 .1 .4 .1 .1466 .344 \\
\hline description & A & 2.5 .4 .13 \\
\hline destinationIndicator & A & 2.5 .4 .27 \\
\hline device & O & 2.5 .6 .14 \\
\hline
\end{tabular}




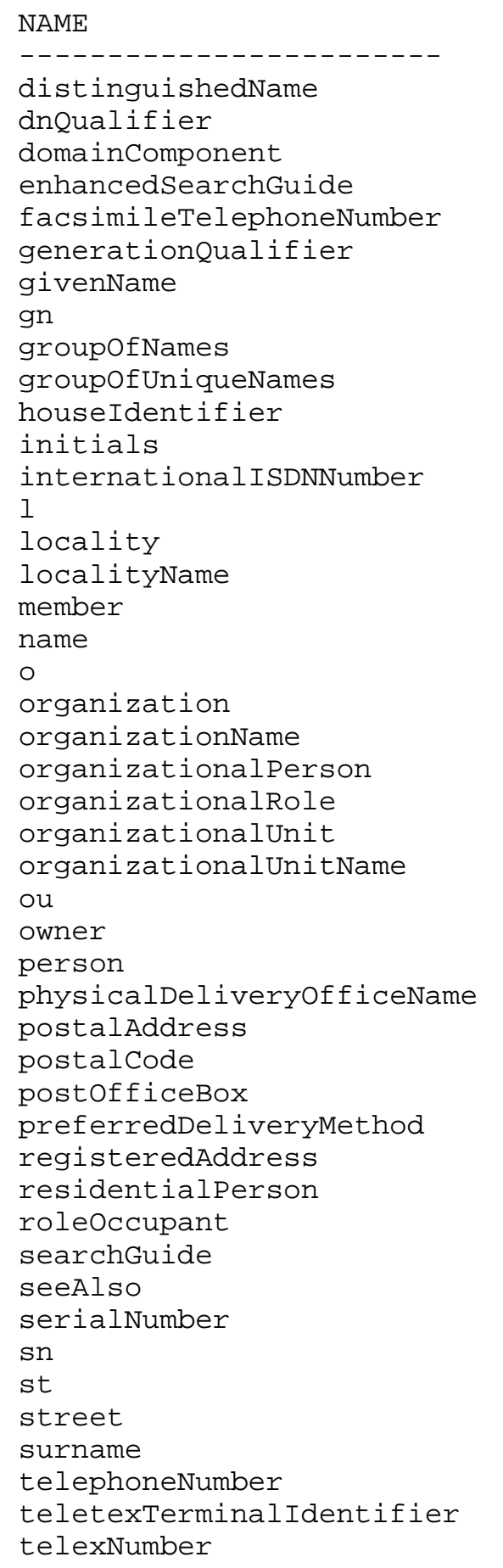

\begin{tabular}{|c|c|}
\hline Type & OID \\
\hline A & 2.5 .4 .49 \\
\hline A & 2.5 .4 .46 \\
\hline A & 0.9 .2342 .19200300 .100 .1 .25 \\
\hline A & 2.5 .4 .47 \\
\hline A & 2.5 .4 .23 \\
\hline A & 2.5 .4 .44 \\
\hline A & 2.5 .4 .42 \\
\hline A & RESERVED \\
\hline O & 2.5 .6 .9 \\
\hline O & 2.5 .6 .17 \\
\hline A & 2.5 .4 .51 \\
\hline A & 2.5 .4 .43 \\
\hline A & 2.5 .4 .25 \\
\hline A & 2.5 .4 .7 \\
\hline O & $2 \cdot 5 \cdot 6 \cdot 3$ \\
\hline A & 2.5 .4 .7 \\
\hline A & 2.5 .4 .31 \\
\hline A & 2.5 .4 .41 \\
\hline A & 2.5 .4 .10 \\
\hline O & 2.5 .6 .4 \\
\hline A & 2.5 .4 .10 \\
\hline O & 2.5 .6 .7 \\
\hline O & 2.5 .6 .8 \\
\hline 0 & 2.5 .6 .5 \\
\hline A & 2.5 .4 .11 \\
\hline A & 2.5 .4 .11 \\
\hline A & 2.5 .4 .32 \\
\hline O & 2.5 .6 .6 \\
\hline A & 2.5 .4 .19 \\
\hline A & 2.5 .4 .16 \\
\hline A & 2.5 .4 .17 \\
\hline A & 2.5 .4 .18 \\
\hline A & 2.5 .4 .28 \\
\hline A & 2.5 .4 .26 \\
\hline 0 & 2.5 .6 .10 \\
\hline A & 2.5 .4 .33 \\
\hline A & 2.5 .4 .14 \\
\hline A & 2.5 .4 .34 \\
\hline A & 2.5 .4 .5 \\
\hline A & 2.5 .4 .4 \\
\hline A & 2.5 .4 .8 \\
\hline A & 2.5 .4 .9 \\
\hline A & 2.5 .4 .4 \\
\hline A & 2.5 .4 .20 \\
\hline A & 2.5 .4 .22 \\
\hline A & 2.5 .4 .21 \\
\hline
\end{tabular}




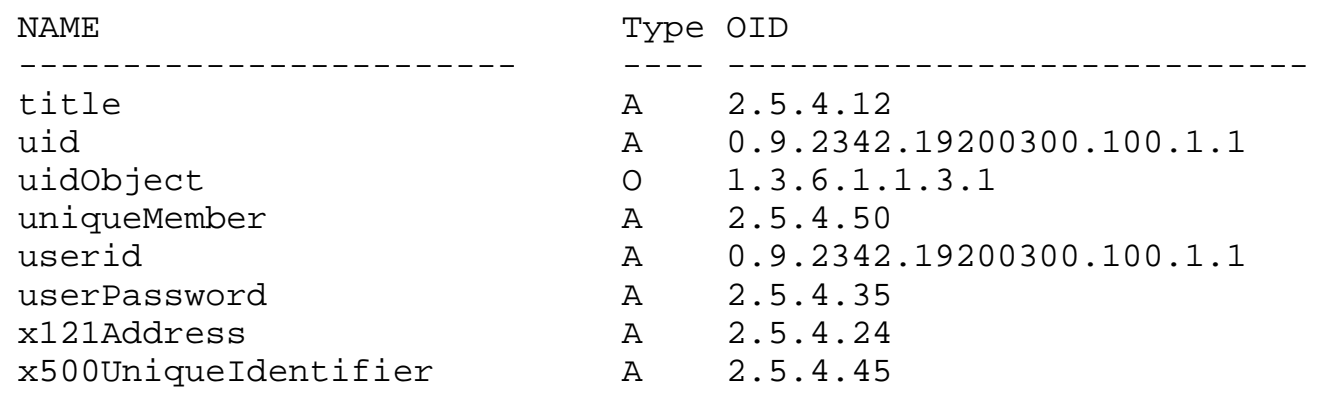

5. Security Considerations

Attributes of directory entries are used to provide descriptive information about the real-world objects they represent, which can be people, organizations, or devices. Most countries have privacy laws regarding the publication of information about people.

Transfer of cleartext passwords is strongly discouraged where the underlying transport service cannot guarantee confidentiality and integrity, since this may result in disclosure of the password to unauthorized parties.

Multiple attribute values for the 'userpassword' attribute need to be used with care. Especially reset/deletion of a password by an administrator without knowing the old user password gets tricky or impossible if multiple values for different applications are present.

Certainly, applications that intend to replace the 'userpassword' value(s) with new value(s) should use modify/replacevalues (or modify/deleteAttribute+addAttribute). In addition, server implementations are encouraged to provide administrative controls that, if enabled, restrict the 'userpassword' attribute to one value.

Note that when used for authentication purposes [RFC4513], the user need only prove knowledge of one of the values, not all of the values.

6. Acknowledgements

The definitions, on which this document is based, have been developed by committees for telecommunications and international standards.

This document is an update of RFC 2256 by Mark Wahl. RFC 2256 was a product of the IETF ASID Working Group. 
The 'dc' attribute type definition and the 'dcobject' object class definition in this document supersede the specification in RFC 2247 by S. Kille, M. Wahl, A. Grimstad, R. Huber, and S. Sataluri.

The 'uid' attribute type definition in this document supersedes the specification of the 'userid' in RFC 1274 by P. Barker and S. Kille and of the uid in RFC 2798 by M. Smith.

The 'uidobject' object class definition in this document supersedes the specification of the 'uidobject' in RFC 2377 by A. Grimstad, R. Huber, S. Sataluri, and M. Wahl.

This document is based upon input of the IETF LDAPBIS working group. The author wishes to thank $S$. Legg and $K$. Zeilenga for their significant contribution to this update. The author would also like to thank Kathy Dally, who edited early versions of this document.

7. References

7.1. Normative References

[E.123] Notation for national and international telephone numbers, ITU-T Recommendation E.123, 1988

[E.164] The international public telecommunication numbering plan, ITU-T Recommendation E.164, 1997

[F.1] Operational Provisions For The International Public Telegram Service Transmission System, CCITT Recommendation $\mathrm{F} .1,1992$

[F.31] Telegram Retransmission System, CCITT Recommendation F.31, 1988

[ISO3166] ISO 3166, "Codes for the representation of names of countries".

[RFC1034] Mockapetris, P., "Domain names - concepts and facilities", STD 13, RFC 1034, November 1987.

[RFC1123] Braden, R., "Requirements for Internet Hosts - Application and Support", STD 3, RFC 1123, October 1989.

[RFC2119] Bradner, S., "Key words for use in RFCs to Indicate Requirement Levels", BCP 14, RFC 2119, March 1997.

[RFC2181] Elz, R. and R. Bush, "Clarifications to the DNS Specification", RFC 2181, July 1997. 
[RFC3490] Faltstrom, P., Hoffman, P., and A. Costello,

"Internationalizing Domain Names in Applications (IDNA)", RFC 3490, March 2003.

[RFC4013] Zeilenga, K., "SASLprep: Stringprep Profile for User Names and Passwords", RFC 4013, February 2005.

[RFC4234] Crocker, D. and P. Overell, "Augmented BNF for Syntax Specifications: ABNF", RFC 4234, October 2005.

[RFC4510] Zeilenga, K., Ed., "Lightweight Directory Access Protocol (LDAP): Technical Specification Road Map", RFC 4510, June 2006 .

[RFC4512] Zeilenga, K., "Lightweight Directory Access Protocol (LDAP): Directory Information Models", RFC 4512, June 2006 .

[RFC4517] Legg, S., Ed., "Lightweight Directory Access Protocol (LDAP): Syntaxes and Matching Rules", RFC 4517, June 2006.

[X.121] International numbering plan for public data networks, ITU-T Recommendation X.121, 1996

[X.509] The Directory: Authentication Framework, ITU-T Recommendation X.509, 1993

[X.520] The Directory: Selected Attribute Types, ITU-T Recommendation X.520, 1993

[X.521] The Directory: Selected Object Classes. ITU-T Recommendation X.521, 1993

\subsection{Informative References}

[RFC1274] Barker, P. and S. Kille, "The COSINE and Internet X.500 Schema", RFC 1274, November 1991.

[RFC2247] Kille, S., Wahl, M., Grimstad, A., Huber, R., and S. Sataluri, "Using Domains in LDAP/X.500 Distinguished Names", RFC 2247, January 1998.

[RFC2377] Grimstad, A., Huber, R., Sataluri, S., and M. Wahl, "Naming Plan for Internet Directory-Enabled Applications", RFC 2377, September 1998.

[RFC2798] Smith, M., "Definition of the inetorgPerson LDAP Object Class", RFC 2798, April 2000. 
[RFC4513] Harrison R., Ed., "Lightweight Directory Access Protocol (LDAP): Authentication Methods and Security Mechanisms", RFC 4513, June 2006.

[RFC4523] Zeilenga, K., "Lightweight Directory Access Protocol (LDAP) Schema Definitions for X.509 Certificates", RFC 4523, June 2006 .

[RFC4524] Zeilenga, E., Ed., "COSINE LDAP/X.500 Schema", RFC 4524, June 2006 .

[X.500] ITU-T Recommendations X.500 (1993) | ISO/IEC 9594-1:1994, Information Technology - Open Systems Interconnection The Directory: Overview of concepts, models and services. 
Appendix A. Changes Made Since RFC 2256

This appendix lists the changes that have been made from RFC 2256 to RFC 4519.

This appendix is not a normative part of this specification, which has been provided for informational purposes only.

1. Replaced the document title.

2. Removed the IESG Note.

3. Dependencies on RFC 1274 have been eliminated.

4. Added a Security Considerations section and an IANA Considerations section.

5. Deleted the conformance requirement for subschema object classes in favor of a statement in [RFC4517].

6. Added explanation to attribute types and to each object class.

7. Removed Section 4, Syntaxes, and Section 6, Matching Rules, (moved to [RFC4517]).

8. Removed the certificate-related attribute types: authorityRevocationList, cACertificate, certificateRevocationList, crosscertificatePair, deltaRevocationList, supportedAlgorithms, and userCertificate.

Removed the certificate-related object Classes: certificationAuthority, certificationAuthority-V2, CRLDistributionPoint, strongAuthenticationUser, and userSecurityInformation

LDAP PKI is now discussed in [RFC4523].

9. Removed the dmdName, knowledgeInformation, presentationAddress, protocolinformation, and supportedApplicationcontext attribute types and the dmd, applicationEntity, and dSA object classes.

10. Deleted the aliasedobjectName and objectClass attribute type definitions. Deleted the alias and top object class definitions. They are included in [RFC4512]. 
11. Added the 'dC' attribute type from RFC 2247, making the distinction between 'stored' and 'query' values when preparing IDN strings.

12. Numerous editorial changes.

13. Removed upper bound after the SYNTAX oid in all attribute definitions where it appeared.

14. Added text about Unicode, SASLprep [RFC4013], and UTF-8 for userpassword.

15. Included definitions, comments and references for 'dcobject' and 'uidobject'.

16. Replaced PKI schema references to use RFC 4523.

17. Spelt out and referenced ABNF on first usage.

18. Removed Section 2.4 (Source). Replaced the source table with explicit references for each definition.

19. All references to an attribute type or object class are enclosed in single quotes.

20. The layout of attribute type definitions has been changed to provide consistency throughout the document:

$>$ Section Heading

$>$ Description of Attribute type

$>$ Multivalued description

$>$ Source Information

$>$ Definition

$>$ Example

$>$ Additional Comments

Adding this consistent output included the addition of examples to some definitions.

21. References to alternate names for attributes types are provided with a reference to where they were originally specified.

22. Clarification of the description of 'distinguishedName' and 'name', in regards to these attribute types being supertypes.

23. Spelt out ISDN on first usage. 
24. Inserted a reference to [RFC4517] for the

'teletexTerminalIdentifier' definition's SYNTAX OID.

25. Additional names were added to the IANA Considerations. Names include 'commonName', 'dcObject' , 'domainComponent' , 'GN' , ' localityName', 'organizationName', 'organizationUnitName', 'surname', 'uidobject' and 'userid'.

26. Renamed all instances of supercede to supersede.

27. Moved [F.1], [F.31] and [RFC4013] from informative to normative references.

28. Changed the ' $C$ ' definition to be consistent with X.500.

Author's Address

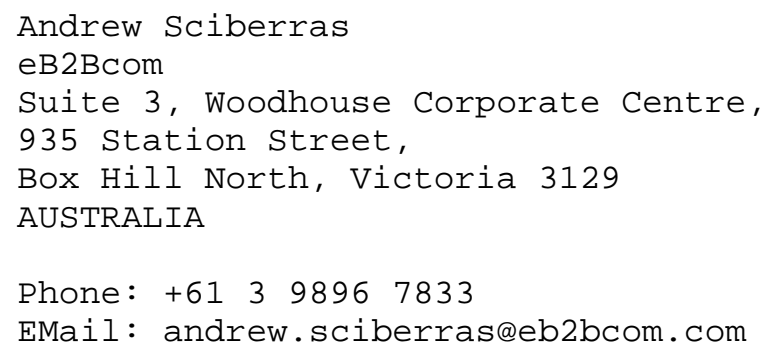


Full Copyright statement

Copyright (C) The Internet Society (2006).

This document is subject to the rights, licenses and restrictions contained in BCP 78, and except as set forth therein, the authors retain all their rights.

This document and the information contained herein are provided on an "AS IS" basis and THE CONTRIBUTOR, THE ORGANIZATION HE/SHE REPRESENTS OR IS SPONSORED BY (IF ANY), THE INTERNET SOCIETY AND THE INTERNET ENGINEERING TASK FORCE DISCLAIM ALL WARRANTIES, EXPRESS OR IMPLIED, INCLUDING BUT NOT LIMITED TO ANY WARRANTY THAT THE USE OF THE INFORMATION HEREIN WILL NOT INERINGE ANY RIGHTS OR ANY IMPLIED WARRANTIES OF MERCHANTABILITY OR FITNESS FOR A PARTICULAR PURPOSE.

Intellectual Property

The IETF takes no position regarding the validity or scope of any Intellectual Property Rights or other rights that might be claimed to pertain to the implementation or use of the technology described in this document or the extent to which any license under such rights might or might not be available; nor does it represent that it has made any independent effort to identify any such rights. Information on the procedures with respect to rights in RFC documents can be found in BCP 78 and BCP 79 .

Copies of IPR disclosures made to the IETF Secretariat and any assurances of licenses to be made available, or the result of an attempt made to obtain a general license or permission for the use of such proprietary rights by implementers or users of this specification can be obtained from the IETF on-line IPR repository at http://www.ietf.org/ipr.

The IETF invites any interested party to bring to its attention any copyrights, patents or patent applications, or other proprietary rights that may cover technology that may be required to implement this standard. Please address the information to the IETF at ietf-ipreietf.org.

Acknowledgement

Funding for the RFC Editor function is provided by the IETF Administrative Support Activity (IASA) . 